\title{
NORMALITY CRITERIA CONCERNING SHARING HOLOMORPHIC FUNCTIONS
}

\author{
FENG LÜ
}

\begin{abstract}
In this paper, we study the problem of normal families of meromorphic functions that share holomorphic functions with their linear differential polynomials. Meanwhile, some results about normal family are derived which improve and generalize several related theorems obtained by Chang, Fang and Zalcman [2], Pang [5] and Schwick [9].
\end{abstract}

\section{Introduction and main results}

In order to state our main results, we need the following definitions and notations.

Let $f, g$ and $a, b$ be holomorphic functions on a domain $D$ in $\mathbb{C}$. We denote the condition that $f(z)-a(z)=0$ implies $g(z)-b(z)=0$ by $f(z)=a(z) \Rightarrow g(z)=b(z)$. If $f(z)=a(z) \Rightarrow g(z)=$ $b(z)$ and $g(z)=b(z) \Rightarrow f(z)=a(z)$, we write $f(z)=a(z) \Leftrightarrow g(z)=b(z)$. Moreover, we say that $f$ and $g$ share $a$ provide that $f(z)=a(z) \Leftrightarrow g(z)=a(z)$. In what follows, we assume that the reader is familiar with the basic notation and results in the Nevanlinna value distribution theory (see, $[11,12])$.

One important subject in the theory of normal family is to find the normal families. According to Bloch's principle, a lot of normality criteria have been obtained by starting from Picard type theorems [8].

In 1992, Schwick [9] drawn a connection between values shared by functions in $\mathscr{F}$ and the normality of the family $\mathscr{F}$. In fact, he proved the following results.

Theorem A. Let $\mathscr{F}$ be a family of meormophic functions in a domain $D$, and let $a_{1}, a_{2}, a_{3}$ be three distinct complex numbers. If for each $f \in \mathscr{F}, f$ and $f^{\prime}$ share $a_{j}(j=1,2,3)$, then $\mathscr{F}$ is normal in $D$.

From then on, many normality criteria have been obtained in this direction (see, $[1,3,4$, 6, 7, 10]). In 2000, Pang and Zalcman [5] obtained the following result.

2010 Mathematics Subject Classification. 30D45, 30D35.

Key words and phrases. Holomorphic function, Normal family, Nevanlinna theory, Linear differential polynomial.

The work was supported by the NSFC Tianyuan Mathematics Youth Fund(11026146). 
Theorem B. Let $\mathscr{F}$ be a family of meormophic functions in a domain $D$, and let $a, b, c$ and $d$ be complex numbers such that $c \neq a$ and $d \neq b$. If for each $f \in \mathscr{F}, f=a \Leftrightarrow f^{\prime}=b$ and $f=c \Leftrightarrow f^{\prime}=d$, then $\mathscr{F}$ is normal in $D$.

In 2005, Chang, Fang and Zalcman [2] replaced the condition $f=c \Leftrightarrow f^{\prime}=d$ by $f=c \Rightarrow$ $f^{\prime}=d$ and improved Theorem $\mathrm{B}$ as follows.

Theorem C. Let $\mathscr{F}$ be a family of meormorphic functions in a domain D, and let $a, b, c$ and $d$ be complex numbers such that $b \neq 0, c \neq a$ and $d \neq b$. If for each $f \in \mathscr{F}, f=a \Leftrightarrow f^{\prime}=b$ and $f=c \Rightarrow f^{\prime}=d$, then $\mathscr{F}$ is normal in $D$.

Remark 1. As a matter of fact, if the condition $d \neq b$ is omitted, Theorem B and C still hold.

From Theorem C, some questions are proposed as follows.

Question 1. Can the values $a, b, c$ and $d$ be weakened to holomorphic functions?

Question 2. Can $f^{\prime}$ be extended to a linear differential polynomial in $f$ ?

In the following, we use the notation

$$
L[f]=a_{0} f^{\prime}+a_{1} f
$$

for a linear differential polynomial in $f$, where $a_{0}, a_{1}$ are two holomorphic functions with $a_{0}(z) \neq 0$.

In the paper, by considering the above questions, we obtain a result as follows, which is an improvement of the precious theorems.

Theorem 1.1. Let $\mathscr{F}$ be a family of meormorphic functions in a domain $D$, let $L[f]$ be defined as (1.1), and let $a, b, c, d$ be four holomorphic functions in $D$. For each $f \in \mathscr{F}$, if

(1) $a(z) \neq c(z)$;

(2) $b(z)-a_{1}(z) a(z)-a_{0}(z) c^{\prime}(z) \neq 0$;

(3) $b(z)-a_{1}(z) c(z)-a_{0}(z) c^{\prime}(z) \neq 0$;

(4) $f(z)=a(z) \Leftrightarrow L[f](z)=b(z)$ and $f(z)=c(z) \Rightarrow L[f](z)=d(z)$, then $\mathscr{F}$ is normal in $D$.

Suppose that $a_{0}=1$ and $a_{1}=0$ in (1.1), we find that the condition (2) coincides with (3) in Theorem 1.1, and both of them reduce to $b(z) \neq c^{\prime}(z)$. So, the following corollary is an immediately consequence of Theorem 1.1.

Corollary 1.2. Let $\mathscr{F}$ be a family of meormorphic functions in a domain $D$, let $a, b, c, d$ be four holomorphic functions in D. For each $f \in \mathscr{F}$, if 
(1) $a(z) \neq c(z)$;

(2) $b(z) \neq c^{\prime}(z)$;

(3) $f(z)=a(z) \Leftrightarrow f^{\prime}(z)=b(z)$ and $f(z)=c(z) \Rightarrow f^{\prime}(z)=d(z)$, then $\mathscr{F}$ is normal in $D$.

Remark 2. If the functions $a, b, c, d$ reduces to constants, then Corollary 1.2 becomes Theorem C. In [2, Theorem 2], the authors given an example to show the necessary of the condition $b \neq 0$, which also shows the necessary of the assumption (3) in Theorem 1.1 and (2) in Corollary 1.2. Now, we state the example here.

Example. Let

$$
f_{n}(z)=\frac{(n z)^{2}}{(n z)^{2}-1}, n=1,2, \cdots
$$

and let $\mathscr{F}=\left\{f_{n}\right\}_{n=1}^{\infty}, D=\{z:|z|<1\}$. Then

$$
f_{n}^{\prime}(z)=\frac{-2 n^{2} z}{\left[(n z)^{2}-1\right]^{2}} .
$$

Clearly, if $f \in \mathscr{F}, f$ and $f^{\prime}$ vanish only at 0 , also $f \neq 1$. Thus, we have $f(z)=0 \Leftrightarrow f^{\prime}(z)=0$ and $f(z)=1 \Rightarrow f^{\prime}(z)=d$ for any $d$. However, $\mathscr{F}$ is not normal in $D$.

Remark 3. For families of holomorphic functions, Theorem 1.1 still valid if the condition (2) is deleted. The proofs of our results have roots in [2].

\section{The lemma}

To prove our result, we need the following lemma, which is essential to our proofs.

Lemma 2.1 ([7]). Let $\mathscr{F}$ be a family of functions meromorphic in the unit disc $\Delta$, all of whose zeros have multiplicity at least $k$, and suppose that there exists $A \geq 1$ such that $\left|f^{(k)}(z)\right| \leq A$ whenever $f(z)=0$. If $\mathscr{F}$ is not normal at $z_{0}$ in the unit disc, then there exist, for each $0 \leq \alpha \leq k$

(a) points $z_{n} \in \Delta, z_{n} \rightarrow z_{0}$;

(b) functions $f_{n} \in \mathscr{F}$ and

(c) positive number $\rho_{n} \rightarrow 0$ such that $\rho_{n}^{-\alpha} f_{n}\left(z_{n}+\rho_{n} \xi\right)=g_{n}(\xi) \rightarrow g(\xi)$ locally uniformly, where $g$ is a non-constant meromorphic function in $\mathbb{C}$, all of whose zeros have multiplicity at least $k$, such that $g^{\sharp}(\xi) \leq g^{\sharp}(0)=k A+1$. In particular, $g$ has order at most two.

\section{Proof of the Theorem 1.1}

Since normality is a local property, we assume that $D=\Delta$, the unit disc. Set $\mathscr{G}=\{F$ : $F=f-c, f \in \mathscr{F}\}$. In view of the form of $L[f]$ and the assumption, we can easily deduce $F(z)=0 \Rightarrow F^{\prime}(z)=\phi(z)$, where

$$
\phi=\frac{d-a_{1} c-a_{0} c^{\prime}}{a_{0}} .
$$


Suppose that $\mathscr{F}$ is not normal at $z_{0} \in \Delta$. We have $A=\max _{z \in D_{1}}\{|\phi(z)|\}$, where $r>0$ is a constant and $D_{1}=\left\{z:\left|z-z_{0}\right| \leq r\right\} \subset \Delta$. Then, in domain $D_{2}=\left\{z:\left|z-z_{0}\right|<\frac{r}{2}\right\}$, we have $F(z)=0 \Rightarrow\left|F^{\prime}(z)\right|=|\phi(z)| \leq M$, where

$$
M=\max \{A, 1\} \geq 1 .
$$

Obviously, $\mathscr{G}$ is not normal at $z_{0}$ as well. Then, by Lemma 1 , we can find a sequence of functions $F_{n} \in \mathscr{G}$, a sequence of complex numbers $z_{n} \rightarrow z_{0}$ and a sequence of positive numbers $\rho_{n} \rightarrow 0$, such that

$$
g_{n}(\xi)=\rho_{n}^{-1} F_{n}\left(z_{n}+\rho_{n} \xi\right)=\rho_{n}^{-1}\left[f_{n}\left(z_{n}+\rho_{n} \xi\right)-c\left(z_{n}+\rho_{n} \xi\right)\right] \rightarrow g(\xi)
$$

converges locally uniformly in $\mathbb{C}$, where $g$ is a non-constant meromorphic function, which satisfies $g^{\sharp}(\xi) \leq g^{\sharp}(0)=M+1$.

In the following, we claim that

(I) $g(\xi)=0 \Rightarrow g^{\prime}(\xi)=\phi\left(z_{0}\right)$,

(II) $g^{\prime}(\xi) \neq B$,

(III) $g(\xi) \neq \infty$,

where $B=\frac{b\left(z_{0}\right)-a_{0}\left(z_{0}\right) c^{\prime}\left(z_{0}\right)-a_{1}\left(z_{0}\right) c\left(z_{0}\right)}{a_{0}\left(z_{0}\right)} \neq 0$ is a constant.

We prove the claim as follows.

By (3.1), we have

$$
g_{n}^{\prime}(\xi)=f_{n}^{\prime}\left(z_{n}+\rho_{n} \xi\right)-c^{\prime}\left(z_{n}+\rho_{n} \xi\right) \rightarrow g^{\prime}(\xi) .
$$

Furthermore

$$
\begin{aligned}
& \frac{L\left[f_{n}\right]\left(z_{n}+\rho_{n} \xi\right)}{a_{0}\left(z_{n}+\rho_{n} \xi\right)}=f_{n}^{\prime}\left(z_{n}+\rho_{n} \xi\right)+\frac{a_{1}\left(z_{n}+\rho_{n} \xi\right)}{a_{0}\left(z_{n}+\rho_{n} \xi\right)} f_{n}\left(z_{n}+\rho_{n} \xi\right) \\
= & f_{n}^{\prime}\left(z_{n}+\rho_{n} \xi\right)+\frac{a_{1}\left(z_{n}+\rho_{n} \xi\right)}{a_{0}\left(z_{n}+\rho_{n} \xi\right)}\left[\rho_{n} g(\xi)+c\left(z_{n}+\rho_{n} \xi\right)\right] \\
\rightarrow & g^{\prime}(\xi)+c^{\prime}\left(z_{0}\right)+\frac{a_{1}\left(z_{0}\right)}{a_{0}\left(z_{0}\right)} c\left(z_{0}\right) .
\end{aligned}
$$

Suppose that $g\left(\eta_{0}\right)=0$. Note that $g \neq 0$, by Hurwitz's theorem and (3.1), there exists a sequence $\eta_{n} \rightarrow \eta_{0}$ such that (for $n$ large enough)

$$
f_{n}\left(z_{n}+\rho_{n} \eta_{n}\right)=c\left(z_{n}+\rho_{n} \eta_{n}\right)
$$


Since $f(z)=c(z) \Rightarrow L[f](z)=d(z)$, we have $L\left[f_{n}\right]\left(z_{n}+\rho_{n} \eta_{n}\right)=d\left(z_{n}+\rho_{n} \eta_{n}\right)$. Then, it follows from (3.3) that

$$
\begin{aligned}
g^{\prime}\left(\eta_{0}\right) & =\lim _{n \rightarrow \infty} \frac{L\left[f_{n}\right]\left(z_{n}+\rho_{n} \eta_{n}\right)}{a_{0}\left(z_{n}+\rho_{n} \eta_{n}\right)}-c^{\prime}\left(z_{0}\right)-\frac{a_{1}\left(z_{0}\right)}{a_{0}\left(z_{0}\right)} c\left(z_{0}\right) \\
& =\lim _{n \rightarrow \infty} \frac{d\left(z_{n}+\rho_{n} \eta_{n}\right)}{a_{0}\left(z_{n}+\rho_{n} \eta_{n}\right)}-c^{\prime}\left(z_{0}\right)-\frac{a_{1}\left(z_{0}\right)}{a_{0}\left(z_{0}\right)} c\left(z_{0}\right) \\
& =\frac{d\left(z_{0}\right)-a_{0}\left(z_{0}\right) c^{\prime}\left(z_{0}\right)-a_{1}\left(z_{0}\right) c\left(z_{0}\right)}{a_{0}\left(z_{0}\right)}=\phi\left(z_{0}\right),
\end{aligned}
$$

which implies (I).

With (3.3), we deduce

$$
\begin{aligned}
& \frac{L\left[f_{n}\right]\left(z_{n}+\rho_{n} \xi\right)-b\left(z_{n}+\rho_{n} \xi\right)}{a_{0}\left(z_{n}+\rho_{n} \xi\right)} \rightarrow g^{\prime}(\xi)+c^{\prime}\left(z_{0}\right)+\frac{a_{1}\left(z_{0}\right)}{a_{0}\left(z_{0}\right)} c\left(z_{0}\right)-\frac{b\left(z_{0}\right)}{a_{0}\left(z_{0}\right)} \\
= & g^{\prime}(\xi)-\frac{b\left(z_{0}\right)-a_{0}\left(z_{0}\right) c^{\prime}\left(z_{0}\right)-a_{1}\left(z_{0}\right) c\left(z_{0}\right)}{a_{0}\left(z_{0}\right)}=g^{\prime}(\xi)-B .
\end{aligned}
$$

Suppose that $g^{\prime}\left(\theta_{0}\right)=B$, obviously $g^{\prime} \neq B$. Otherwise $g^{\prime}=B$ and $g(\xi)=B \xi+C$, where $C$ is a constant. From (I), we deduce $B=\phi\left(z_{0}\right)$. Then, a simple calculation shows that

$$
M+1=g^{\sharp}(0) \leq\left|\phi\left(z_{0}\right)\right|<M+1,
$$

which is a contradiction.

Then, by Hurwitz's theorem and (3.4), there exists a sequence $\theta_{n} \rightarrow \theta_{0}$, such that (for $n$ sufficiently large)

$$
L\left[f_{n}\right]\left(z_{n}+\rho_{n} \theta_{n}\right)-b\left(z_{n}+\rho_{n} \theta_{n}\right)=0
$$

From the assumption, we have $f_{n}\left(z_{n}+\rho_{n} \theta_{n}\right)=a\left(z_{n}+\rho_{n} \theta_{n}\right)$. Then, by (3.1) and $a(z) \neq c(z)$, we derive that

$$
\begin{aligned}
g\left(\theta_{0}\right) & =\lim _{n \rightarrow \infty} \rho_{n}^{-1}\left[f_{n}\left(z_{n}+\rho_{n} \rho_{n}\right)-c\left(z_{n}+\rho_{n} \rho_{n}\right)\right] \\
& =\lim _{n \rightarrow \infty} \rho_{n}^{-1}\left[a\left(z_{n}+\rho_{n} \eta_{n}\right)-c\left(z_{n}+\rho_{n} \eta_{n}\right)\right]=\infty,
\end{aligned}
$$

a contradiction. Thus, we prove (II).

Now, we prove (III). Suppose that $g\left(\xi_{0}\right)=\infty$. Since $g \neq \infty$, there exists a closed disc $K=$ $\left\{\xi:\left|\xi-\xi_{0}\right| \leq \delta\right\}$ on which $1 / g$ and $1 / g_{n}$ are holomorphic (for $n$ large enough) and $1 / g_{n} \rightarrow 1 / g$ uniformly. From $a(z) \neq c(z)$, we deduce

$$
\frac{1}{g_{n}(\xi)}-\frac{\rho_{n}}{a\left(z_{n}+\rho_{n} \xi\right)-c\left(z_{n}+\rho_{n} \xi\right)} \rightarrow \frac{1}{g(\xi)}
$$

uniformly on $K$. Assume that the multiplicity of the zero of $1 / g$ at $\xi_{0}$ be $m$. Then, $\left(\frac{1}{g}\right)^{(m)}\left(\xi_{0}\right) \neq$ 0 . Noting that $1 / \mathrm{g}$ is not a constant, then, by Hurwitz's theorem and (3.5), there exist $\xi_{n, j} \rightarrow$ 
$\xi_{0}(j=1,2 \cdots, m)$ such that

$$
\frac{1}{g_{n}\left(\xi_{n, j}\right)}-\frac{\rho_{n}}{a\left(z_{n}+\rho_{n} \xi_{n, j}\right)-c\left(z_{n}+\rho_{n} \xi_{n, j}\right)}=0 .
$$

It is not difficult from (3.6) to deduce

$$
f_{n}\left(z_{n}+\rho_{n} \xi_{n, j}\right)=a\left(z_{n}+\rho_{n} \xi_{n, j}\right),(j=1,2 \cdots, m) .
$$

Thus, we have

$$
L\left[f_{n}\right]\left(z_{n}+\rho_{n} \xi_{n, j}\right)=b\left(z_{n}+\rho_{n} \xi_{n, j}\right) .
$$

Observing that the form of $L[f]$, we derive

$$
f_{n}^{\prime}\left(z_{n}+\rho_{n} \xi_{n, j}\right)=\left.\frac{b-a_{1} a}{a_{0}}\right|_{z=z_{n}+\rho_{n} \xi_{n, j}} .
$$

Furthermore,

$$
\begin{aligned}
g_{n}^{\prime}\left(z_{n}+\rho_{n} \xi_{n, j}\right) & =f_{n}^{\prime}\left(z_{n}+\rho_{n} \xi_{n, j}\right)-c^{\prime}\left(z_{n}+\rho_{n} \xi_{n, j}\right) \\
& =\left.\frac{b-a_{1} a-a_{0} c^{\prime}}{a_{0}}\right|_{z=z_{n}+\rho_{n} \xi_{n, j}},(j=1,2 \cdots, m) .
\end{aligned}
$$

It follows from $b(z)-a_{1}(z) a(z)-a_{0}(z) c^{\prime}(z) \neq 0$ that, for $j=1,2 \cdots, m$,

$$
\left.\left(\frac{1}{g_{n}}\right)^{\prime}\right|_{\xi=\xi_{n, j}}=-\frac{g_{n}^{\prime}\left(\xi_{n, j}\right)}{g_{n}^{2}\left(\xi_{n, j}\right)}=-\left.\rho_{n}^{2} \frac{b-a_{1} a-a_{0} c^{\prime}}{(a-c)^{2}}\right|_{z=z_{n}+\rho_{n} \xi_{n, j}} \neq 0 .
$$

That is $\xi_{n, j} \neq \xi_{n, i}(1 \leq i \neq j \leq m)$. Set

$$
H_{n}(\xi)=\left(\frac{1}{g_{n}(\xi)}\right)^{\prime}+\left.\rho_{n}^{2} \frac{b-a_{1} a-a_{0} c^{\prime}}{(a-c)^{2}}\right|_{z=z_{n}+\rho_{n} \xi} .
$$

For $j=1,2 \cdots, m$, we have $H_{n}\left(\xi_{n, j}\right)=0$ and

$$
H_{n}(\xi) \rightarrow\left(\frac{1}{g(\xi)}\right)^{\prime}
$$

uniformly on $K$. Combining (3.10), Hurwitz's theorem and $H_{n}\left(\xi_{n, j}\right)=0(j=1,2 \cdots, m)$ yields that $\xi_{0}$ is a zero of $\left(\frac{1}{g}\right)^{\prime}$ with multiplicity $m$, and thus $\left(\frac{1}{g}\right)^{(m)}\left(\xi_{0}\right)=0$, which is a contradiction. Thus, we prove (III).

Hence, we finish the proof of the claim.

It follows from (III) that $g$ is an entire function and therefore of exponential type. By (II), we have

$$
g^{\prime}(\xi)=B+\lambda \mu e^{\mu \xi}
$$


where $\lambda(\neq 0), \mu$ are two constant. Suppose that $\mu=0$, then $g^{\prime}=B$. From $g^{\sharp}(0)=M+1$, it is easy to deduce a contradiction. Now, we assume that $\mu \neq 0$. Integral (3.11) yields

$$
g(\xi)=B \xi+D+\lambda e^{\mu \xi}
$$

where $D$ is a constant. Let $\beta_{0}$ be a zero of $g(\xi)$. It means that

$$
B \beta_{0}+D+\lambda e^{\mu \beta_{0}}=0
$$

By (I), we have $g^{\prime}\left(\beta_{0}\right)=\phi\left(z_{0}\right)$. That is

$$
B+\lambda \mu e^{\mu \beta_{0}}=\phi\left(z_{0}\right)
$$

Combining (3.13) and (3.14) yields $\beta_{0}=\frac{B-\phi\left(z_{0}\right)-D \mu}{\mu B}$, which indicates that $g$ has the unique zero $\beta_{0}$. But it is obvious from (3.12) that $g$ has infinitely many zeros.

Thus, $\mathscr{G}$ is normal at $z_{0}$, and $\mathscr{F}$ is normal at $z_{0}$ as well. Furthermore, $\mathscr{F}$ is normal in $D$. Hence, we complete the proof of the Theorem 1.1.

\section{References}

[1] H. H. Chen and M. L. Fang, Shared values and normal families of meromorphic functions, J. Math. Anal. Appl. 260(2001), 124-132.

[2] J. M. Chang, M. L. Fang and L. Zalcman, Normality and fixed-points of meromorphic functions, Ark. Mat. 43(2005), 307-321.

[3] M. L. Fang and Y. Xu, Normal families of holomorphic functions and shared values, Israel J. Math. 129 (2002), 125-141.

[4] M. L. Fang and L. Zalcman, Normal families and shared values of meromorphic functions, Ann. Polon. Math. 80(2003), 133-141.

[5] X. C. Pang, Shared values and normal families, Analysis 22(2002), 175-182.

[6] X. C. Pang and L. Zalcman, Normality and shared values, Ark. Mat. 38(2000), 171-282.

[7] X. C. Pang and L. Zalcman, Normal families and shared values, Bull. London Math. Soc. 32(2000), 325-331.

[8] J. Schiff, Normal families, Springer-Verlag, New York/Berlin, 1993.

[9] W. Schwick. Sharing values and normality, Arch. Math. 59 (1992), 50-54.

[10] Y. Xu, Normality criteria concerning sharing values, Indian J. Pure Appl. Math. 30 (1999), 287-293.

[11] L. Yang, Value distribution theory, Springer-Verlag \& Science Press, Berlin, 1993.

[12] H.X. Yi and C.C. Yang, Uniqueness theory of meromorphic functions, Science Press, Beijing, 1995.

College of Science, China University of Petroleum, Qingdao 266580, Shandong, P. R. China.

E-mail: lvfeng18@gmail.com 